\title{
It's Good to be Flexible: Energy Transport Facilitated by Conformational Fluctuations in Light-Harvesting Polymers.
}

Leah M. Rader Bowers, ${ }^{1}$ Egle Puodziukynaite, ${ }^{2,3}$ Li Wang, ${ }^{1}$ Zachary A. Morseth, ${ }^{1}$ Kirk S. Schanze, ${ }^{2}$ John R. Reynolds, ${ }^{3}$ and John M. Papanikolas ${ }^{1^{*}}$

${ }^{1}$ Department of Chemistry, University of North Carolina at Chapel Hill, Chapel Hill, NC, 27599, United States. ${ }^{2}$ Department of Chemistry, University of Texas at San Antonio, San Antonio, TX, 78249, United States, United States.

${ }^{3}$ School of Chemistry and Biochemistry, School of Materials Science and Engineering, Center for Organic Photonics and Electronics, Georgia Institute of Technology, Atlanta, GA 30332, United States.

Energy tranfer efficiency was calculated using the ground-state emission spectrum of the PF- $\mathrm{Ru}_{60} \mathrm{Os}_{10}$ polymer shown in Figure $\mathrm{S} 1 \mathrm{~A}$. The spectra was scaled to account for the difference in quantum yield between Ru andn Os models $\left(\Phi_{\mathrm{Ru}}=7 \%\right.$ and $\Phi_{\mathrm{Os}}=0.32 \%$, respectively) so the emission represents number of photons emitted not emission intensity (Figure S1B). Efficiency is determined to be $\sim 80-85 \%$ by dividing the integrated area under the Os emission curve by the total area under the Ru and Os complex emission curves.

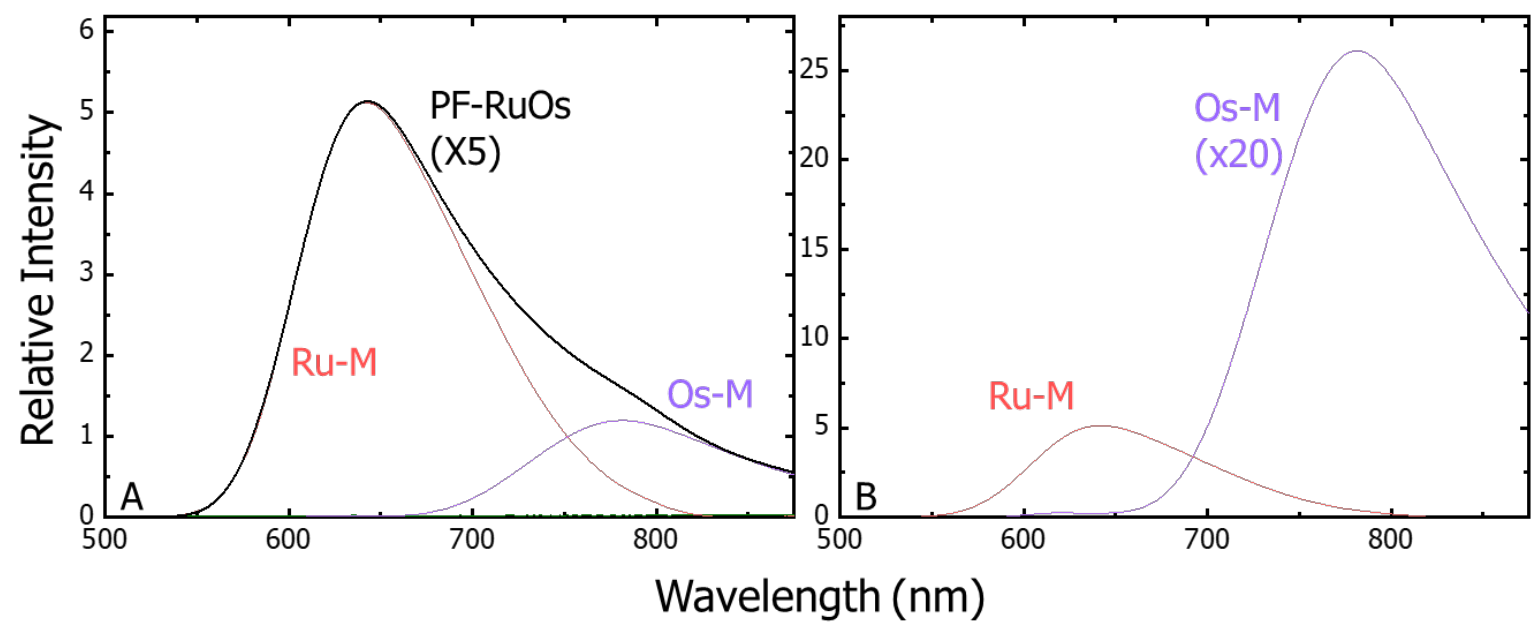

Figure S1. Ground state emission spectrtum of A) PF-Ru60 $\mathrm{Os}_{10}$ (black) with spectra of $\mathrm{Ru}$ and Os model complexes (Ru-M, red; Os$\mathrm{M}$, purple, respectively) superimposed and B) spectra of Ru-M and scaled Os-M emission.

The all-atom MD PF-Ru simulation is run for $40 \mathrm{~ns}$ on a cell where all components are represented as atoms in their pre-selected groups (Figure S2). While the simulation cell remains all-atom in nature, pre-selected grouping of atoms allows us to visualize them as future beads and make measurments between their centers of mass. 


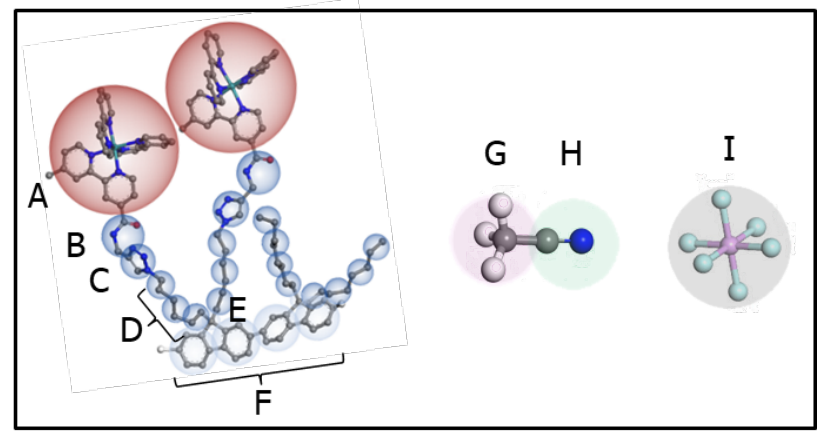

Figure S2. Representation of attoms in pre-selected groups A through I.

In order to apply an appropriate bond stretch interaction potential between each bound bead pair in the CG model, we measure equilibrium bond lengths $\left(R_{0}\right)$ between those pre-selected groups (Equation 2A). We list all bound pre-selected group sets in the first column of Table S1. Descriptions of atoms in groups A through I are shown in Table S1.

\begin{tabular}{|c|c|}
\hline Bead Type & Atoms \\
\hline A & $-\mathrm{C}_{31} \mathrm{H}_{25} \mathrm{~N}_{6}-\mathrm{Ru}$ \\
\hline $\mathrm{B}$ & $-\mathrm{CO}-\mathrm{NH}-\mathrm{CH}_{2}$ \\
\hline $\mathrm{C}$ & $-\mathrm{C}-\mathrm{N}_{3}-\mathrm{CH}$ \\
\hline $\mathrm{D}$ & $-\mathrm{CH}_{2}-\mathrm{CH}_{2}$ or $-\mathrm{CH}_{2}-\mathrm{CH}_{3}$ \\
\hline $\mathrm{E}$ & $-\mathrm{C}$ \\
\hline $\mathrm{F}$ & - Ph or $-\mathrm{Ph}-\mathrm{C}$ \\
\hline G & $-\mathrm{CH}_{3}$ \\
\hline $\mathrm{H}$ & $-\mathrm{CN}$ \\
\hline I & $\mathrm{PF}_{6}$ \\
\hline
\end{tabular}

Table S1. Description of atoms in pre-selectetd groups A through I. 


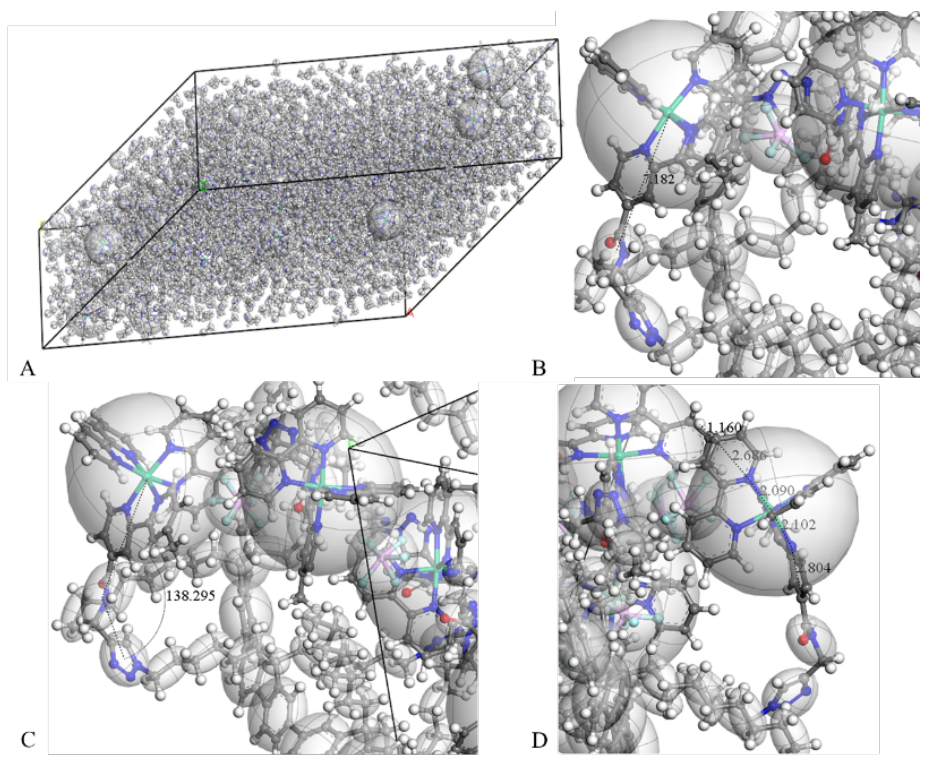

Figure S3. Measurements in the all-atom MD PF-Ru 20mer model. A) All-atom simulation cell packed with PF-Ru, MeCN solvent and $\left[\mathrm{PF}_{6}\right]^{-}$counter ions represented in their pre-selected groups. B) Measuring across bonds to determine the size of pre-selected group A. C) Measuring the angle between pre-selected groups A, B and C. D) Measuring bond length between pre-selected groups.

Measurements for each parameter highlighted in Tables S1-4 were taken at every monomeric segment of the PFRu 20 structure. For example, equilibrium angles were determined between three pre-selected group centers along each pendant and backbone monomeric unit. The distributions were obtained by sampling at at $2 \mathrm{fs}$ intervals out to $20 \mathrm{~ns}$, and over all equivalent angles in each of the monomer units.

\begin{tabular}{ccc}
\hline Bead Type Set & $\begin{array}{c}\text { Equilibrium Bond Length, } \\
R_{0}(\AA)\end{array}$ & $\begin{array}{c}\text { Force Constant, } k_{B} \\
\left(\mathrm{kcal} / \mathrm{mol} / \AA^{2}\right)\end{array}$ \\
\hline A - B & 7.72 & 2.99 \\
B - C & 4.06 & 2.99 \\
C - D & 3.26 & 2.99 \\
D - E & 3.99 & 2.99 \\
D - D & 3.08 & 2.99 \\
E - F & 4.53 & 2.99 \\
F - F & 4.22 & 2.99 \\
G - H & 2.04 & 2.99 \\
\hline
\end{tabular}

Table S2. Bond stretch potential function parameters.

To determine $R_{0}$ between $\mathrm{A}$ and $\mathrm{B}(\mathrm{A}-\mathrm{B})$, for example, we start the all-atom simulation at $\mathrm{t}=0$ ns and measure distances between all pre-selected group A and B pairs (Figure S2B). We extract all A - B distances at each time step out to 40 ns, average the resulting data, and find the equilibrium bond distance is $7.72 \AA$ (Table S2). This type of measurement is repeated for all pre-selected group 
sets. We use values from Table SI 2 along with a uniform $k_{B}$ of $2.99 \mathrm{kcal} / \mathrm{mol} / \AA^{2}$ to calculate bonding interaction potentials (Equation 2A) in CG MD simulations.

A similar process is used to measure the angle force constant $\left(k_{A}\right)$ and equilibrium bond angle $\left(\theta_{0}\right)$ between all pre-selected groups. Values we get from these measurements allow us to apply an appropriate angle bending interaction potential between each bound bead set in the CG model (Equation 2B).

\begin{tabular}{ccc}
\hline Bead Type Set & $\begin{array}{r}\text { Force Constant, } k_{A} \\
(\mathrm{kcal} / \mathrm{mol})\end{array}$ & Equilibrium Angle, $\theta_{0}\left(^{0}\right)$ \\
\hline A - B - C & 60 & 126 \\
B - C - D & 8 & 154 \\
C - D - D & 7 & 140 \\
D - D - D & 12 & 180 \\
D - D - E & 60 & 120 \\
D - E - D & 8 & \\
\hline
\end{tabular}

Table S3. Angle bend potential function parameters.

In Figure S2C, we show an example of how angle A, B and C (A - B - C) is measured. We select all angles A - B - C at t $=0$ ns and extract the data across the trajectory. Instead of simply averaging the data, we fit our results to Equation $2 \mathrm{~B}$ and pull out constants for $k_{A}$ and $\theta_{0}$ that provide the best fits (Figure S4). We find that set $\mathrm{A}-\mathrm{B}-\mathrm{C}$ forms an equilibrium angle of $126^{\circ}$ with a force constant of $60 \mathrm{kcal} / \mathrm{mol}$ and repeat this type of measurement and fitting procedure for all pre-selected group sets (Table SI3).

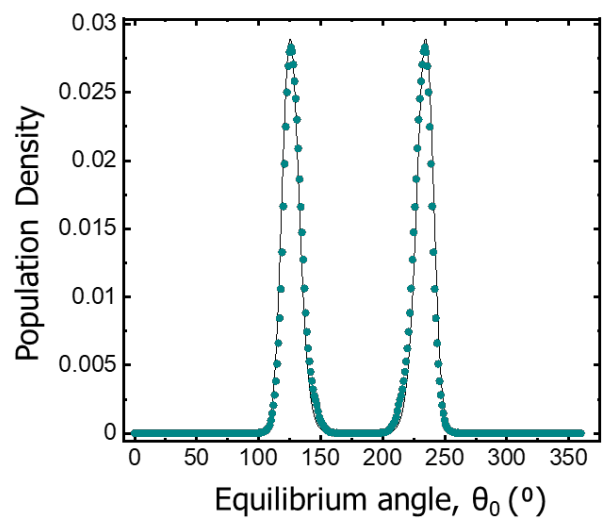

Figure S4. Fitting of angle distribution.

Accurate values for the radius of each pre-selected group $\left(\sigma_{i i}\right)$ and interaction energy between two groups $\left(\varepsilon_{i i}\right)$ are required to parameterize Van der Waals potential interactions between pairs of non-bonding beads in the CG model (Equation 2C). We determine the value for $\sigma_{i i}$ by physically measuring across bonds of a pre-selected group and adding the Lennard Jones radii of atoms at each end. Figure S2D illustrates an example of this measurement for group A. 


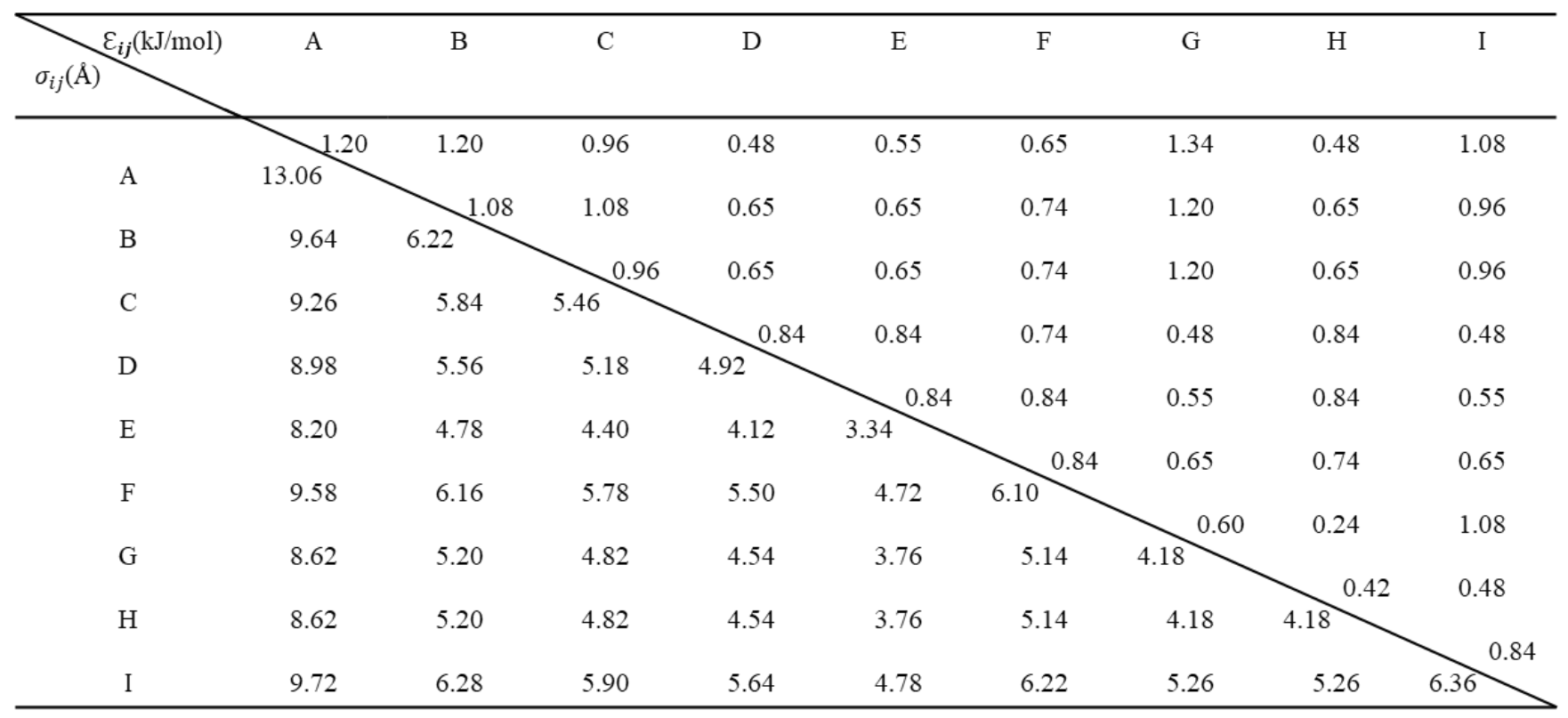

Table S4. Van der Waals potential function parameters.

Under the, the interaction radius of $\mathrm{A}\left(\sigma_{A A}\right)$ is the average of two $\mathrm{A}$ radii. We measure across all pre-selected groups $\mathrm{A}$ at $\mathrm{t}=0 \mathrm{~ns}$, propagate the trajectory, and determine $\sigma_{A A}$ to be $13.06 \AA$ on average (Table S4). We repeat this type of measurement on preselected groups B-F and I. Interaction radii between two different beads is found by calculating their average radii (i.e. $\sigma_{A B}$ ). The sizes of the two beads in the MeCN solvent molecule were adjusted to reproduce the experimentally observed solvent density at room temperature.

The final interaction potential we must parameterize is the Coulombic potential that exists between any two charged or partially charged groups. Unlike the other three interaction potentials, values of charge $\left(q_{i}\right.$ and $\left.q_{j}\right)$, solvent and vacuum permittivity $\left(\varepsilon_{1}\right.$ and $\varepsilon_{0}$, respectively) are found in the literature. In our CG model, we place charges of +2 and -1 at the center of all Ru complexes and $\mathrm{CG}\left[\mathrm{PF}_{6}\right]$ counter ions, respectively, and represent $\mathrm{CG} \mathrm{MeCN}$ as two beads that carry a partial charge of \pm 0.432 to approximate the dipole moment of the solvent. We apply Coulombic interaction potentials to all charged non-bonding bead sets in the CG model.

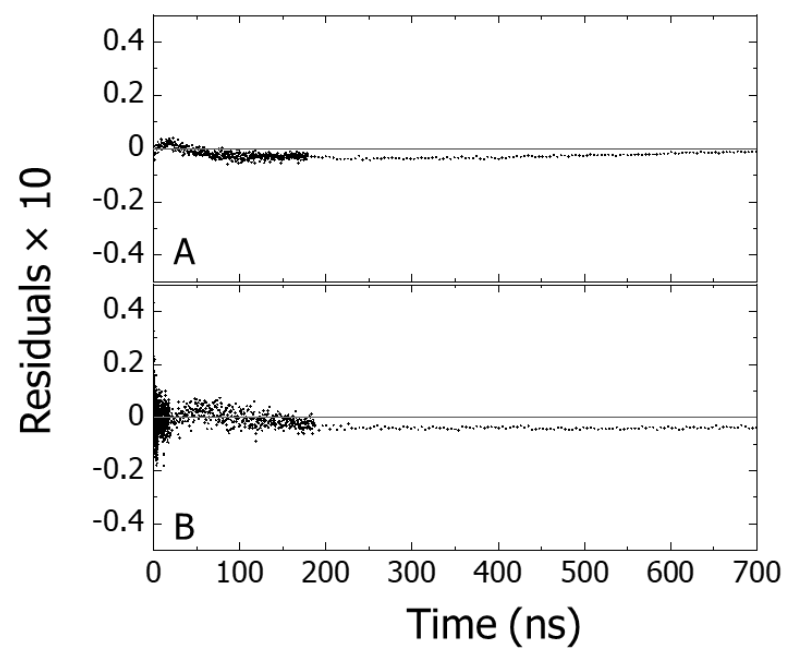

Figure S5. Residual plots of fitting shown in Figure 9. A) The residual when the simulated kinetics of Ru* population is compared to the Ru experimental trace. B) The residual when the simulated kinetics of Os ${ }^{*}$ population is compared to the Os experimental trace. 
Multiple time resolved emission traces were stitched together to maintain high temporal resolution at short times and observe excited state emission out to long times. This creates experimental traces with varied time resolutions compared to the consistent resolution of the simulated time steps ( $40 \mathrm{ps}$ ). To determine the residual between the simulated and experimental trace, time points of the experimental trace were interpolated with the time points given by the simulation. 